\title{
Value of preoperative neutrophil-to-lymphocyte ratio in predicting thyroid cancer in intermediate category fine needle aspiration pathology: retrospective study
}

\section{Yasar Ozdenkaya}

Istanbul Medipol Universitesi

\section{Pelin Basim}

Istanbul Medipol Universitesi

Oktay Olmuscelik

Istanbul Medipol Universitesi

Naciye Cigdem Arslan ( $\sim$ cigdemarslan@hotmail.it)

Istanbul Medipol Universitesi https://orcid.org/0000-0002-2282-7207

Research article

Keywords: Goiter, Bethesda classification, fine needle aspiration biopsy, papillary thyroid carcinoma, thyroid nodule, neutrophil-to-lymphocyte ratio

Posted Date: November 20th, 2019

DOI: https://doi.org/10.21203/rs.2.17529/v1

License: (9) This work is licensed under a Creative Commons Attribution 4.0 International License. Read Full License 


\section{Abstract}

Background The management of thyroid nodules with intermediate category of the Bethesda System for Reporting Thyroid Cancer (TBSRTC) is still debatable. Aim of this study is to investigate the diagnostic accuracy of neutrophil-to-lymphocyte ratio (NLR) in detecting cancer in intermediate TBSRTC categories.

Methods Data of the patients who underwent thyroidectomy between 2012 and 2018 were analyzed retrospectively. Demographic characteristics, complete blood count, TSH value, TBSRTC categories and postoperative pathology of the patients were collected. The association with preoperative NLR and postoperative pathology was assessed.

Results Of 146 patients included in the study, 57 (39\%) were in TBSRTC III, IV and V category. The mean NLR in this subgroup was $2.1 \pm 0.8$ and similar between TBSRTC III, IV and IV groups ( $p=0.737)$. Thyroid cancer was detected in $38(66.7 \%)$ patients. The mean NLR was $2.4 \pm 0.8$ in thyroid cancers and significantly higher when compared with patients with benign postoperative pathology $(1.6 \pm 0.4$, $p=0.014)$. There was a significant difference between papillary and follicular cancer; the mean NLR was $2.3 \pm 0.8$ in papillary cancer and $2.6 \pm 0.7$ in follicular cancer patients $(p=0.005)$. The cut-off value of NLR for predicting cancer at $89 \%$ sensitivity and $53 \%$ specificity was 1.6 (Area under curve: $0.769,95 \%$ confidence interval: $0.643-0.895, p=0.001$ ).

Conclusions Preoperative NLR is elevated in patients with thyroid cancer in intermediate TBSRTC categories. Despite low diagnostic accuracy, considering its availability and low costs, NLR has merit for further studies.

\section{Background}

During last decades, the incidence of thyroid cancer has been nearly tripled from 4.9 to 14.3 per 100,000 people in United States due to increasing diagnosis of thyroid nodules[1]. The major issue in evaluation of thyroid pathologies is distinguishing benign and malign nodules. Up to $15 \%$ of all clinically detected thyroid nodules are found to be malignant [2]. Ultrasonography and fine needle aspiration biopsy (FNAB) are the most common diagnostic tools with high specificity to detect thyroid cancer, however one-third of the cytologic evaluation cannot provide a certain diagnosis.

In 2007, The Bethesda System for Reporting Thyroid Cytopathology (TBSRTC) has been introduced to standardize a category-based reporting system for thyroid FNAB including 6 categories, each indicating an implied cancer risk [3]. According to the 2017 update for TBSRTC system, 20-30\% of the cases are reported as intermediate category with reported rates of cancer $10-30 \%$ for TBSRTC III, $25-40 \%$ for TBSRTC IV and 50-75\% for TBSRTC V [3-5]. Several studies have validated the risk stratification of TBSRTC and the increase in diagnostic value of FNAB with the use of TBRTC [6-9]. Particularly in intermediate categories, Bethesda system has limited diagnostic accuracy $[7,10,11]$. Repeating FNAB [3], lobectomy [3], sonographic risk stratification $[5,10]$ and molecular tests [12] have been recommended for more accurate preoperative staging in TBRTC III, IV and V categories, however these tools come with 
potential interventional complications and increased costs. These concerns lead to focus on more feasible additional tools to improve preoperative risk assessment $[5,12]$.

The systemic inflammatory response is considered to promote different stages of cancer development and invasion. Several studies reported that elevated preoperative NLR is associated with deeper tumor invasion, advanced stage, and poor prognosis in different tumors [13-16]. The significance of NLR in detecting thyroid papillary microcarcinomas was first reported in 2013 [17]. After that many studies have suggested that NLR, as a marker of systemic inflammation, is related with tumor characteristics and prognosis in thyroid cancer [18-20]. Neutrophil-to-lymphocyte ratio may be an easily accessible and inexpensive tool for preoperative decision-making particularly in patients with conflicting preoperative FNAB results.

Aim of this study is to investigate the diagnostic accuracy of preoperative NLR for predicting cancer in TBSRTC III, IV and IV thyroid nodules.

\section{Methods}

An institutional board-approved database of thyroidectomy was reviewed. The data of the patients who underwent bilateral thyroidectomy for thyroid nodules between 2012 and 2018 were retrospectively analyzed. Bethesda classification has been put into practice in 2012 in our institution. Patients who had available laboratory tests, FNAB reports and TBSRTC classification were included in the study. Neutrophil to lymphocyte ratio was calculated from routine preoperative laboratory tests. The impedance method (Beckman Coulter LH 750) was used for complete blood count. All the patients underwent FNAB and thyroidectomy at the same institution. American Thyroid Association (ATA) guidelines were followed in the management of thyroid nodules [21]. Biopsies were performed by radiologists under ultrasound guidance. None of the specimens were on-site evaluated for adequacy. Two experienced pathologists evaluated the specimens at institutional pathology laboratory. The cytologic evaluation was reported according to current TBSRTC classifications [3]. Patients who did not have preoperative complete blood count within 1 week before the surgery, FNAB reports which were not available or did not report Bethesda classification and patients who had signs of thyroiditis, immunosuppression; chemotherapy history or any infectious process within 2 weeks before the surgery were excluded from the analysis.

The primary endpoint of the study was assessing the diagnostic accuracy of preoperative NLR for detecting thyroid cancer in patients who had intermediate (TBSRTC III, IV and V) preoperative FNAB reports.

\section{Statistical analysis}

SPSS for Windows 21.0 (IBM, IL, USA) was used for statistics. Continuous variables were expressed as means and standard deviation and categorical variables as frequency and percentages. The equality of distribution of NLR between variables was tested by Bonferroni analysis. Association between NLR and 
categorical variables was tested by independent samples $t$ test and One-way ANOVA. Pearson's correlation test was used for analyzing association between NLR and continuous variables. Receiver operative characteristic curves were used to calculate the diagnostic accuracy parameters of NLR. Statistical significance was defined as $p<0.05$.

\section{Results}

Between 2012 and 2018, 220 patients underwent thyroidectomy for thyroid nodules in our institution. The reason for exclusion was lack of proper pathology reports in 33 patients, blood count in 19 patients, and informed consent in 12 patients. Ten patients had thyroiditis and were not included in the analysis.

Totally 146 patients were included in the study. The mean age was $45.9 \pm 13.6,109$ (74.7\%) patients were female and 37 (25.3\%) were male. The mean NLR was 2.1 \pm 1.1 . Postoperative pathologic examination revealed thyroid cancer in $92(63 \%)$ of the patients. Details of the demographic and clinical characteristics, Bethesda system and postoperative pathology are given in Table 1.

The TBSRTC classification was intermediate in $57(39 \%)$ patients. The mean NLR in this subgroup was $2.1 \pm 0.8$. The mean NLR was similar between TBSRTC III, IV and IV groups ( $p=0.737)$ (Table 2$)$. Thyroid cancer was detected in postoperative pathologic evaluation in $38(66.7 \%)$ patients. The mean NLR was $2.4 \pm 0.8$ in thyroid cancers and significantly higher when compared with patients with benign postoperative pathology $(1.6 \pm 0.4, p=0.014)$. There was a significant difference between papillary and follicular cancer; the mean NLR was $2.3 \pm 0.8$ in papillary cancer and $2.6 \pm 0.7$ in follicular cancer patients $(p=0.005)$. The cut-off value of NLR for predicting cancer at $89 \%$ sensitivity and $53 \%$ specificity was 1.6 (Area under curve [AUC]: 0.769, 95\% confidence interval [CI]: 0.643-0.895, $p=0.001$ ) (Figure 1). The accuracy of NLR for different cut-off values is given in Table 3.

\section{Discussion}

The Bethesda classification alone may fail in preoperative decision making in intermediate risk category thyroid biopsies [11]. Different complimentary adjunct diagnostic tools including molecular testing and imaging modalities have been suggested to enhance the confidence of preoperative diagnosis. However, there are limited and conflicting data regarding the relationship between preoperative inflammatory biomarkers and thyroid cancer. We found increased preoperative NLR values in patients who were preoperatively in intermediate stages of Bethesda but diagnosed with cancer after surgery. This increase was more remarkable in follicular cancer. Our results showed that preoperative elevated NLR was associated with higher risk of thyroid cancer in intermediate TBSRTC categories.

Elevated preoperative NLR has been shown to be related with incomplete response to therapy [22], poor tumor differentiation [23], lymph node invasion [24], advanced stage [25] and higher thyroglobulin values [26] in thyroid malignancies. On the other hand, in 2016, a meta-analysis of 6 prospective cohorts with 6283 differentiated thyroid cancer patients revealed no difference in NLR between patients with cancer and benign nodules with a mean difference of $0.19(95 \% \mathrm{Cl}:-0.09-0.46, p<0.001)$. This meta-analysis 
concluded that an elevated NLR could not predict differentiated thyroid cancer, however well-designed large studies were needed [27].

In 2017, Manatakis et al [28] reported poor clinicopathological characteristics including extrathyroidal invasion $(p=0.04)$, bilateral $(p=0.03)$ and multifocal tumors $(p=0.01)$ and lymph node metastasis $(P=$ 0.03 ) in thyroid papillary cancer patients with higher NLR. The NLR value was not influenced by sex, age, tumor size or cytology in this study. Conformably with these findings, NLR was not associated with age ( $p$ $=0.795)$, sex $(p=0.076)$, TSH $(0.385)$, FNAB results $(p=0.863)$ and histologic subtype $(p=0.096)$ in our total series, however in subgroup analysis of intermediate TBSRTC categories NLR was significantly higher in follicular cancer $(p=0.005)$. The diagnostic accuracy of systemic inflammatory markers have been evaluated in a recent study including 397 patients by the same group [29]. They found significantly higher NLR $(p=0.026)$ in carcinomas and microcarcinomas compared to benign nodules, but similar platelet-to-lymphocyte ratio values between cancer and benign pathology. The diagnostic accuracy of NLR was $45-50 \%$ in their study. In our study accuracy ranging between 51 to $74 \%$ was higher than previous studies but still unsatisfying. Our analysis focused on the presence of cancer in intermediate Bethesda stages, therefore we did not assess the relationship between NLR and prognostic clinicopathologic factors. Other major drawback of our study is limited number of cases.

\section{Conclusion}

The recent guideline of ATA [21] recommends that molecular tests and radiology can be used to support cytology in cases with indeterminate FNAB, pointing out that the positive and negative predictive values of all diagnostic tools are influenced by the individual cancer rate in each cytologic category, which varies widely at different institutions [12]. There is no doubt that none of the diagnostic tolls alone can provide a certain recommendation of follow-up or surgery. Patients in this 'gray zone' should be evaluated individually with all cytologic and radiologic findings to determine the cancer risk. Despite limited and conflicting evidence in the literature, preoperative NLR is an internationally available, fast and easy measurement which can be suggestive when discussing the management, thus should be kept noteworthy for further studies.

\section{Abbreviations}

TBSRTC: The Bethesda System for Reporting Thyroid Cancer

NLR: Neutrophil-to-lymphocyte ratio

TSH: Thyroid stimulating hormone

FNAB: Ultrasonography and fine needle aspiration biopsy

ATA: American Thyroid Association 
AUC: Area under curve

Cl: Confidence interval

\section{Declarations}

Ethics approval and consent to participate: The study was approved by institutional ethic committee (approval no: 10840098-604.01.01-E.45124). All the patients gave written consent for medical treatments and interventions. All procedures performed in studies involving human participants were in accordance with the ethical standards of the institutional research committee and with the 1964 Helsinki declaration and its later amendments or comparable ethical standards.

Consent for publication: All the patients gave written consent for publication and participating in the study.

Availability of data and material: The data of the patients are available and can be shared on demand.

Competing interests: Authors declare no potential conflict of interests.

Funding: The study received no funding.

Authors' contributions: All authors helped to perform the study; Yasar Ozdenkaya wrote the paper, collected the data, designed the study. Oktay Olmuscelik collected the data and critically revised the manuscript. Pelin Basim collected the histopathological examinations and critically revised the manuscript. Naciye Cigdem Arslan wrote the manuscript, performed the statistical analysis and approved the final revisions.

Acknowledgements: None

\section{References}

[1]L. Davies and H. G. Welch, "Current thyroid cancer trends in the United States," JAMA Otolaryngol. Head Neck Surg., 2014 Apr;140(4):317-22.

[2]J. P. Brito, A. J. Yarur, L. J. Prokop, B. Mclver, M. H. Murad, and V. M. Montori, "Prevalence of Thyroid Cancer in Multinodular Goiter Versus Single Nodule: A Systematic Review and Meta-Analysis," Thyroid, 2013.

[3]E. S. Cibas and S. Z. Ali, "The 2017 Bethesda System for Reporting Thyroid Cytopathology," J. Am. Soc. Cytopathol., vol. 6, no. 6, pp. 217-222, 2017.

[4]M. Bongiovanni, A. Spitale, W. C. Faquin, L. Mazzucchelli, and Z. W. Baloch, "The Bethesda system for reporting thyroid cytopathology: A meta-analysis," Acta Cytologica. 2017;61(2):172. 
[5]R. L. Ferris, Z. Baloch, V. Bernet, et al "Risk stratification of the thyroid nodule with Bethesda indeterminate cytology, category III, IV, V on the one surgeon-performed US-guided fine-needle aspiration with 27-gauge needle, verified by histopathology of thyroidectomy: the additional value of on," J. Am. Soc. Cytopathol., vol. 71, no. 6, pp. 131-136, 2018.

[6]D. Ozdemir, N. Bestepe, S. Faki, A. Kilicarslan, O. Parlak, R. Ersoy, and B. Cakir, "Comparison of thyroid fine needle aspiration biopsy results before and after implementation of Bethesda classification," Cytopathology, 2017 Oct;28(5):400-406.

[7]P. S. Sullivan, S. L. Hirschowitz, P. C. Fung, and S. K. Apple, "The impact of atypia/follicular lesion of undetermined significance and repeat fine-needle aspiration: 5 years before and after implementation of the Bethesda System," Cancer Cytopathol., 2014 Dec;122(12):866-72.

[8]E. Machała, J. Sopiński, I. lavorska, and K. Kołomecki, "Correlation of Fine Needle Aspiration Cytology of Thyroid Gland with Histopathological Results," Polish J. Surg., vol. 90, no. 5, pp. 1-5, 2018.

[9]H. Her-Juing Wu, C. Rose, and T. M. Elsheikh, "The Bethesda system for reporting thyroid cytopathology: An experience of 1,382 cases in a community practice setting with the implication for risk of neoplasm and risk of malignancy," Diagn. Cytopathol., 2012 May;40(5):399-403.

[10]F. F. R. Maia, P. S. Matos, E. J. Pavin, and D. E. Zantut-Wittmann, "Thyroid imaging reporting and data system score combined with Bethesda system for malignancy risk stratification in thyroid nodules with indeterminate results on cytology," Clin. Endocrinol. (Oxf)., 2015 Mar;82(3):439-44.

[11]J. D. Galbraith, N. Swann, B. Cox, N. Letendre, and J. Recabaren, "The bethesda classification for thyroid fine needle aspiration: A predictor or an alarmist?" in American Surgeon, 2018 Jan 1;84(1):161164.

[12]R. L. Ferris, Z. Baloch, V. Bernet, A. Chen, T. J. Fahey, I. Ganly, S. P. Hodak, E. Kebebew, K. N. Patel, A. Shaha, D. L. Steward, R. P. Tufano, S. M. Wiseman, and S. E. Carty, "American Thyroid Association Statement on Surgical Application of Molecular Profiling for Thyroid Nodules: Current Impact on Perioperative Decision Making," Thyroid, vol. 25, no. 7, pp. 760-768, 2015.

[13]H. Yodying, A. Matsuda, M. Miyashita, S. Matsumoto, N. Sakurazawa, M. Yamada, and E. Uchida, "Prognostic Significance of Neutrophil-to-Lymphocyte Ratio and Platelet-to-Lymphocyte Ratio in Oncologic Outcomes of Esophageal Cancer: A Systematic Review and Meta-analysis," Ann. Surg. Oncol., 2016 Feb;23(2):646-54.

[14]H. Tang, W. Lu, B. Li, C. Li, Y. Xu, and J. Dong, “Prognostic significance of neutrophil-to-lymphocyte ratio in biliary tract cancers: a systematic review and meta-analysis," Oncotarget, 2017 May 30;8(22):36857-36868. 
[15]Q. T. Huang, L. Zhou, W. J. Zeng, Q. Q. Ma, W. Wang, M. Zhong, and Y. H. Yu, "Prognostic significance of neutrophil-to-lymphocyte ratio in ovarian cancer: A systematic review and meta-analysis of observational studies," Cellular Physiology and Biochemistry. 2017;41(6):2411-2418.

[16]K. Atila, N. C. Arslan, S. Derici, A. E. Canda, O. Sagol, I. Oztop, and S. Bora, "Neutrophil-to-lymphocyte ratio: Could it be used in the clinic as prognostic marker for gastrointestinal stromal tumor?," Hepatogastroenterology., vol. 61, no. 134, 2014.

[17]C. Seretis, S. Gourgiotis, G. Gemenetzis, F. Seretis, E. Lagoudianakis, and G. Dimitrakopoulos, "The significance of neutrophil/lymphocyte ratio as a possible marker of underlying papillary microcarcinomas in thyroidal goiters: A pilot study," Am. J. Surg., 2013 Jun;205(6):691-6.

[18]J. Y. Kim, T. Park, S. H. Jeong, C. Y. Jeong, Y. T. Ju, Y. J. Lee, S. C. Hong, W. S. Ha, S. K. Choi, and E. J. Jung, "Prognostic importance of baseline neutrophil to lymphocyte ratio in patients with advanced papillary thyroid carcinomas," Endocrine, 2014 Aug;46(3):526-31.

[19]C. L. Liu, J. J. Lee, T. P. Liu, Y. C. Chang, Y. C. Hsu, and S. P. Cheng, "Blood neutrophil-to-lymphocyte ratio correlates with tumor size in patients with differentiated thyroid cancer," J. Surg. Oncol., 2013 Apr;107(5):493-7.

[20]B. H. H. Lang, C. P. C. Ng, K. B. Au, K. P. Wong, K. K. C. Wong, and K. Y. Wan, “Does preoperative neutrophil lymphocyte ratio predict risk of recurrence and occult central nodal metastasis in papillary thyroid carcinoma?," World J. Surg., 2014 Oct;38(10):2605-12.

[21]B. Haugen, E. K. Alexander, K. C. Bible, and et al, "2015 American Thyroid Association Management Guidelines for Adult Patients with Thyroid Nodules and Differentiated Thyroid Cancer," The American Thyroid Association Guidelines Task Force on Thyroid Nodules and Differentiated Thyroid Cancer, 2016 Jan;26(1):1-133.

[22]F. Lee, P.-S. Yang, M.-N. Chien, J.-J. Lee, C.-H. Leung, and S.-P. Cheng, "An Increased Neutrophil-toLymphocyte Ratio Predicts Incomplete Response to Therapy in Differentiated Thyroid Cancer.," Int. J. Med. Sci., 2018 vol. 15, no. 14, pp. 1757-1763.

[23]D. Kocer, C. Karakukcu, H. Karaman, F. Gokay, and F. Bayram, "May the neutrophil/lymphocyte ratio be a predictor in the differentiation of different thyroid disorders?," Asian Pacific J. Cancer Prev., vol. 16, no. 9, pp. 3875-3879, 2015.

[24]N. Xu, Y. Jian, Y. Wang, and W. Tian, "Evaluation of neutrophil-to-lymphocyte ratio and calcitonin concentration for predicting lymph node metastasis and distant metastasis in patients with medullary thyroid cancer.," Mol. Clin. Oncol., 2018v ol. 9, no. 6, pp. 629-634.

[25]W. Gong, S. Yang, X. Yang, and F. Guo, "Blood preoperative neutrophil-to-lymphocyte ratio is correlated with TNM stage in patients with papillary thyroid cancer," Clinics, vol. 71, no. 6, pp. 311-314, 2016. 
[26]S. Ozmen, O. Timur, I. Calik, K. Altinkaynak, E. Simsek, H. Gozcu, A. Arslan, and A. Carlioglu, "Neutrophil-lymphocyte ratio (NLR) and platelet-lymphocyte ratio (PLR) may be superior to C-reactive protein (CRP) for predicting the occurrence of differentiated thyroid cancer," Endocr. Regul., vol. 51, no. 3, pp. 131-136, 2017.

[27]J. F. Liu, L. Ba, H. Lv, D. Lv, J. T. Du, X. M. Jing, N. J. Yang, S. X. Wang, C. Li, and X. X. Li, "Association between neutrophil-to-lymphocyte ratio and differentiated thyroid cancer: A meta-analysis," Sci. Rep. 2016, vol. 6, pp. 1-7.

[28]D. K. Manatakis, S. Tseleni-Balafouta, D. Balalis, V. N. Soulou, D. P. Korkolis, G. H. Sakorafas, G. Plataniotis, and E. Gontikakis, "Association of Baseline Neutrophil-to-Lymphocyte Ratio with Clinicopathological Characteristics of Papillary Thyroid Carcinoma," Int. J. Endocrinol., vol. 2017, 2017:8471235.

[29]D. K. Manatakis, S. Tseleni-Balafouta, L. Tzelves, D. Balalis, A. Tzortzopoulou, D. P. Korkolis, G. H. Sakorafas, E. Gontikakis, and G. Plataniotis, "Diagnostic Accuracy of Preoperative Neutrophil-toLymphocyte and Platelet-to-Lymphocyte Ratios in Detecting Occult Papillary Thyroid Microcarcinomas in Benign Multinodular Goitres," J. Thyroid Res., vol. 2018, pp. 1-6, 2018.

\section{Tables}

Table 1. Demographic and clinical characteristics of the patients and the relationship with neutrophil-to-lymphocyte ratio.

\begin{tabular}{|c|c|c|c|}
\hline & $\mathrm{n}=146$ & NLR (mean \pm SD) & $\mathrm{p}$ \\
\hline Sex $(\mathrm{n}, \%)$ & & & $0.076^{*}$ \\
\hline Female & $109(74.7)$ & $2.1 \pm 1.1$ & \\
\hline Male & $37(25.3)$ & $1.8 \pm 0.5$ & \\
\hline Age (year, mean \pm SD) & $45.9 \pm 13.6$ & & $0.795^{* *}$ \\
\hline TSH (mIU/L, mean \pm SD) & $5.58 \pm 21.4$ & & $0.385^{* *}$ \\
\hline FNAB $(\mathrm{n}, \%)$ & & & $0.863^{* * *}$ \\
\hline TBSRTC I & $3(2.1)$ & $1.8 \pm 0.8$ & \\
\hline TBSRTC II & $50(34.2)$ & $1.9 \pm 1.3$ & \\
\hline TBSRTC III & $15(10.3)$ & $2.2 \pm 0.8$ & \\
\hline TBSRTC IV & $13(8.9)$ & $1.9 \pm 0.9$ & \\
\hline TBSRTC V & $29(19.9)$ & $2 \pm 0.7$ & \\
\hline TBSRTC VI & $36(24.7)$ & $2.1 \pm 0.9$ & \\
\hline Pathology $(\mathrm{n}, \%)$ & & & $0.026^{*}$ \\
\hline Benign & $54(37)$ & $1.8 \pm 0.2$ & \\
\hline Cancer & $92(63)$ & $2.2 \pm 0.8$ & \\
\hline Papillary cancer & $87(59.6)$ & $2.2 \pm 0.8$ & $0.096^{* * *}$ \\
\hline Follicular cancer & $3(2.1)$ & $2.5 \pm 0.9$ & \\
\hline Lymphoma & $1(0.7)$ & 3.1 & \\
\hline Medullary cancer & $1(0.7)$ & 2.4 & \\
\hline Tumor size (mm, mean \pm SD) & $1.9 \pm 1.3$ & & $0.564^{* *}$ \\
\hline
\end{tabular}


NLR: neutrophil-to-lymphocyte ratio, FNAB: Fine needle aspiration biopsy, TBSRTC: The Bethesda System for Reporting Thyroid Cancer, $*$ Independent samples $t$ test, **Pearson correlation, $* * *$ One-way ANOVA

Table 2. Details of the patients with intermediate category fine needle aspiration pathology reports.

\begin{tabular}{|c|c|c|c|}
\hline & $\mathrm{n}=57(\%)$ & NLR (mean \pm SD) & $\mathrm{p}$ \\
\hline FNAB & & & $0.737^{* * *}$ \\
\hline TBSRTC III & $15(26.3)$ & $2.21 \pm 0.8$ & \\
\hline TBSRTC IV & $13(22.8)$ & $2 \pm 0.9$ & \\
\hline TBSRTC V & $29(50.9)$ & $2.1 \pm 0.7$ & \\
\hline Pathology & & & $0.014^{*}$ \\
\hline Benign & $19(33.3)$ & $1.6 \pm 0.4$ & \\
\hline Cancer & $38(66.7)$ & $2.4 \pm 0.8$ & \\
\hline Papillary cancer & 36 & $2.3 \pm 0.8$ & \\
\hline Follicular cancer & 2 & $2.6 \pm 0.7$ & $0.005^{* * *}$ \\
\hline
\end{tabular}

NLR: neutrophil-to-lymphocyte ratio, FNAB: Fine needle aspiration biopsy, TBSRTC: The Bethesda System for Reporting Thyroid Cancer, ***One-way ANOVA, *Independent samples $t$ test

Table 3. Diagnostic accuracy analysis for neutrophil-to-lymphocyte ratio

\begin{tabular}{|c|c|c|c|c|c|c|}
\hline Sensitivity & 95\% CI & Specificity & 95\% CI & PPV & NPV & Accuracy \\
\hline $95 \%$ & $82.2-99.4$ & $31 \%$ & $12.6-56.6$ & $74 \%$ & $75 \%$ & $74 \%$ \\
\hline $89 \%$ & $75.2-97.1$ & $53 \%$ & $28.9-75.6$ & $79 \%$ & $71 \%$ & $77 \%$ \\
\hline $63 \%$ & $45.6-78.2$ & $63 \%$ & $38.4-83.7$ & $77 \%$ & $46 \%$ & $63 \%$ \\
\hline $55 \%$ & $38.3-71.4$ & $90 \%$ & $66.9-98.7$ & $91 \%$ & $50 \%$ & $67 \%$ \\
\hline $29 \%$ & $15.5-45.9$ & $95 \%$ & $73.9-99.9$ & $92 \%$ & $40 \%$ & $51 \%$ \\
\hline
\end{tabular}

\section{Figures}




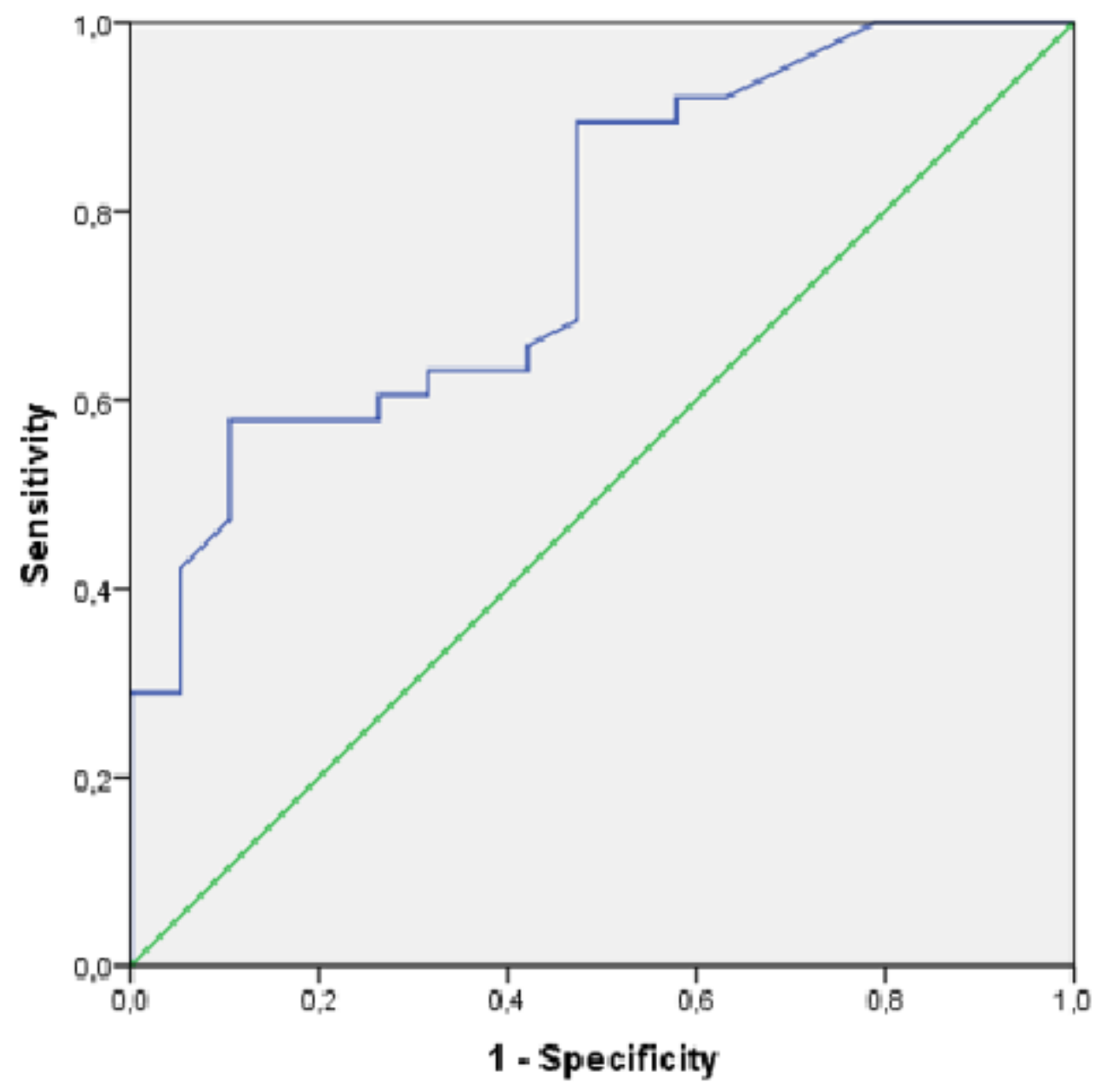

Figure 1

Receiver operative characteristic curve for NLR in predicting thyroid cancer (Area under curve: $0.769,95 \%$ confidence interval: 0.643-0.895, $p=0.001$ ). 